\title{
Downscaling of Spatial Irradiance Based on Cloud Advection using Transfer Functions
}

\author{
Joseph Ranalli \\ Penn State Hazleton \\ Hazleton, PA \\ jar339@psu.edu
}

\author{
Esther E.M. Peerlings \\ German Aerospace Center (DLR), Institute of Networked Energy Systems \\ Oldenburg, Germany \\ Esther.Peerlings@dlr.de
}

\begin{abstract}
Spatiotemporal aggregation of solar irradiance occurs when a spatially distributed receiver (e.g. a PV generation facility) collects variable geographically distributed irradiance and reduces it to a single electrical generation output. Models of this phenomenon exist, and are designed to take variability from a single point irradiance monitor and predict how that variability will be reduced by aggregation. We have applied these models in a revered manner to assess whether the models can be used to predict the variability of a single point measurement given an aggregate irradiance time series as an input. Results for an advection-based model show that this approach leads to overprediction of the high frequency variability due to overprediction of the site-to-site correlation. Incorporating predictions of site pair decorrelation from the wavelet variability model can temper the degree of overprediction and produces more realistic point time series. Further work may be warranted to further improve upon these efforts and enable reliable, transfer function-based downscaling of irradiance data.
\end{abstract}

Index Terms - variability, spatial aggregation, wavelet variability model, transfer function, downscaling

\section{INTRODUCTION}

Spatiotemporal variability of solar irradiance and its characterization are well studied in the literature [1], [2]. It is important to have a good understanding of variability for both photovoltaic (PV) plant management and transmission grid operation in order to anticipate and handle short term ramp events in power generation. One specific aspect of spatiotemporal variability that has been studied in the literature is modeling how variability of a single point sensor is reduced by the aggregation effect associated with collection by a spatially distributed plant [3]-[5].

The Cloud Advection Model (CAM) of Ranalli et al. [5] uses a transfer function to represent the aggregation process. In principle, this approach is reversible. In the present study, we evaluate the ability of the model to be used for the reverse problem of predicting a single point irradiance time series on the basis of a measured spatial aggregate time series. This is similar to the study by Lave and Weekly who used an extrapolated transfer function to generate a high frequency irradiance time series from low resolution data [?]. The CAM solves for the aggregation of a 1-D plant subject to advection of a frozen cloud field over its spatial extent. The CAM represents the plant as a transfer function, which can be written as the Fourier transform of the plant's spatial distribution.

$$
T F(f)=\mathcal{F}\left[d^{*}\left(\frac{x}{V_{c}}\right)\right]
$$

\section{Reverse Aggregation Process}

Given the reversible nature of the transfer function, it is also possible in principle to compute the disaggregation effect, whereby we input an aggregated time signal, $p(t)$, compute the transfer function of the distributed plant, and predict the time series of a single site. This is done mathematically by solving Eq. 2 for $G_{r e f}(f)$ and performing the inverse Fourier transform to yield the single point irradiance time series, $g_{r e f}(t)$.

$$
T F(f)=\frac{P(f)}{G_{r e f}(f)}
$$

We have performed this calculation for an approximately 1-D subset of the HOPE-Melpitz measurement campaign [6], choosing a one hour window during with strong cloud advection was observed. The predicted transfer function for this case is shown in Fig. 1. The transfer function is an overall low-pass filter with some dynamics that allow some bands of high frequency content to pass.

The results of the inverse (disaggregation) transformation for both time series and variability are shown in Fig. 2. The result shows a significant degree of high frequency noise. This is visible both in the time series, and the variability, which is significantly overpredicted for small $\Delta t$. In part, this is due to the fact that the transfer function exhibits near-zero values in its magnitude (see e.g. red curve in Fig. 1), that result in substantial magnification of the oscillations described by the input aggregate power spectrum.

We demonstrate one possible improvement on the calculation by limiting amplification of non-correlated portions of the input. For example, we may see from the figure that the aggregate power spectrum reaches low absolute values at increasing frequency that correspond roughly to the reduction in the coherence shown in Fig. ??. The disaggregated prediction from the CAM instead has a relatively large peak around a frequency of $0.1 \mathrm{~Hz}$, as well as uniformly elevated power spectrum magnitude beyond about $0.2 \mathrm{~Hz}$, which show up as oscillating signal in the time series. As a demonstration of improving the time series, we replaced the 

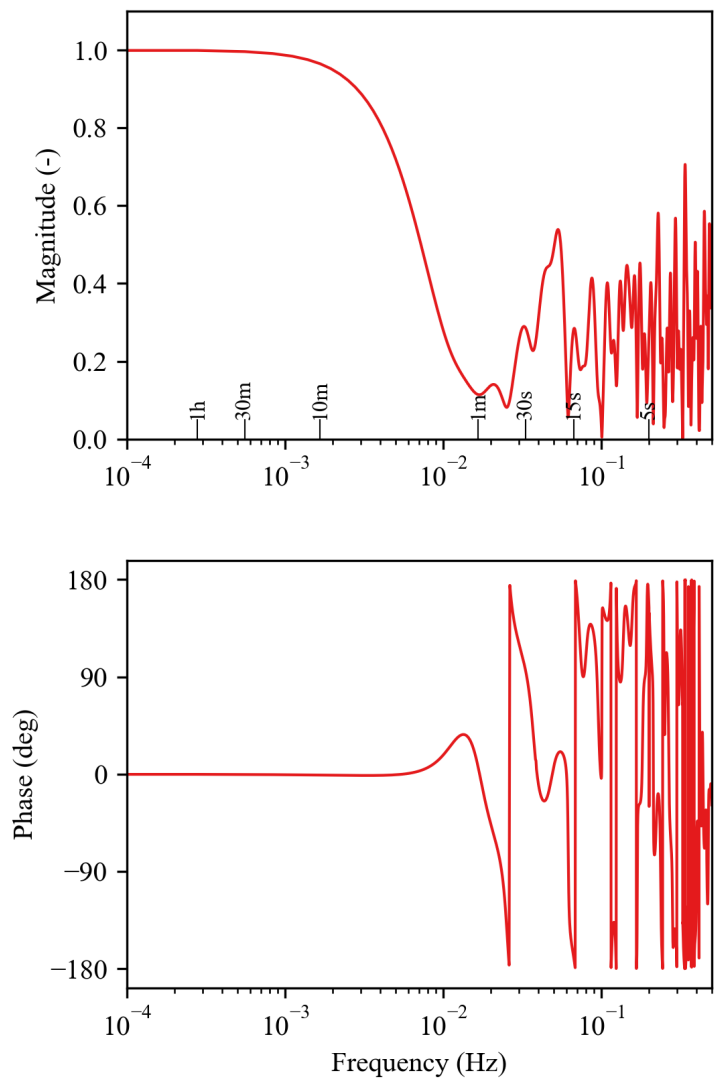

Fig. 1. Predicted transfer function for the plant configuration during the 1 hour window studied here. Several time scales corresponding to the frequencies are labelled.

TABLE I

RMSE FOR CLEAR-SKY INDEX TIME SERIES PREDICTIONS.

\begin{tabular}{ccc} 
& CAM & CAM Modified \\
\hline RMSE & 0.16 & 0.13
\end{tabular}

predicted disaggregated power spectrum values with those of the aggregated power spectrum for values of the aggregate power spectrum less than a threshold (magnitude less than 0.4 in this demonstration). This essentially corrects for the fact that uncorrelated noise is present at high frequencies in the power spectrum, and the assumption of correlation inherent to the transfer function approach is violated. The results of this simplistic correction are shown in Fig. 3. As is evident, this correction removes the high frequency oscillations and creates a somewhat more realistic time series, but the variability predictions are reduced below the target values. Comparisons of the Root Mean Square Error (RMSE) for the time series is provided in Table I, demonstrating the improvement.

While this demonstration suggests the possibility of using the transfer function for disaggregation, results indicate that further research may be needed to fully develop the technique to avoid the amplification of undesirable noise. One limitation
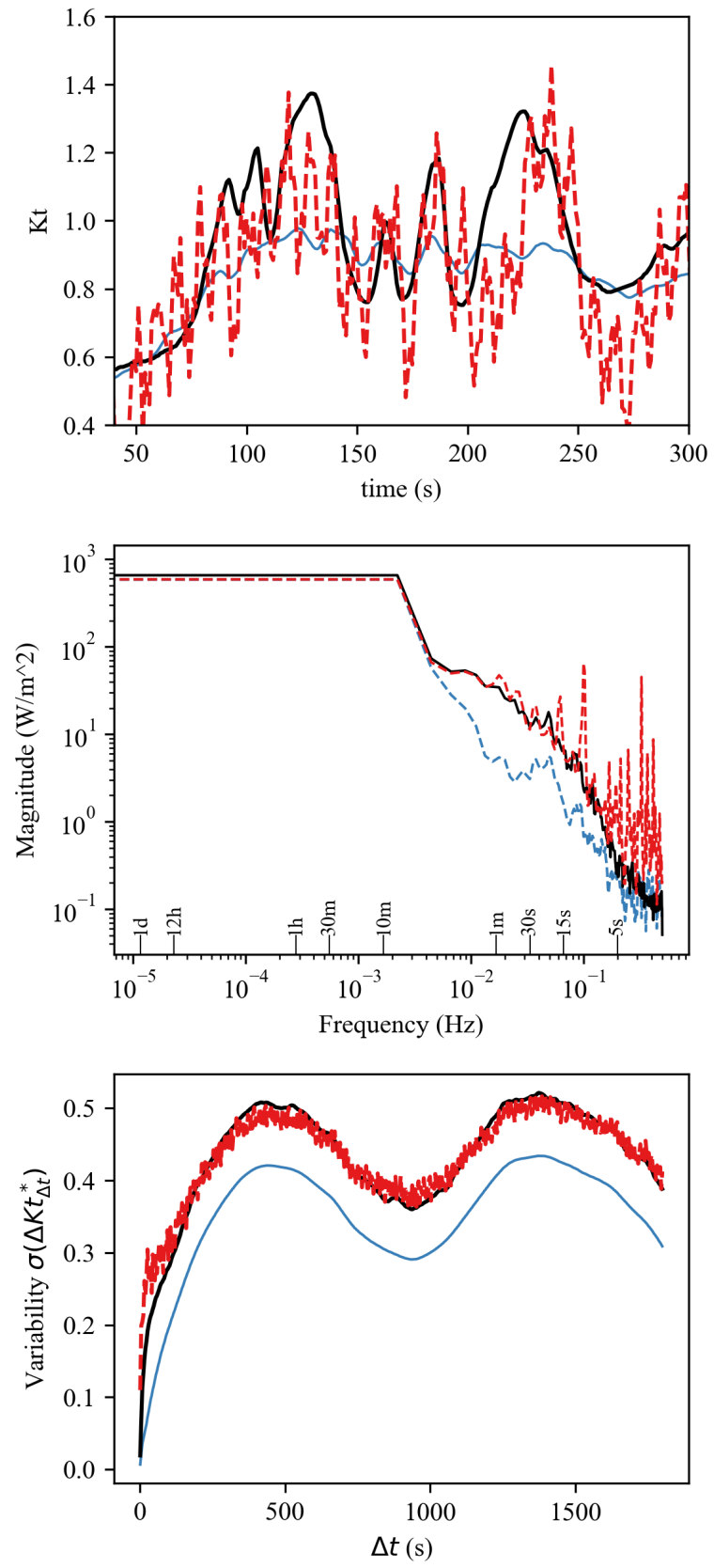

---- aggregate $\longrightarrow$ single site ---- CAM Single

Fig. 2. Disaggregation calculation time series, power spectrum and variability for 9 sites disaggregated to a single reference. Spectra are smoothed using 8 averages with a Hanning window and $50 \%$ overlap. 

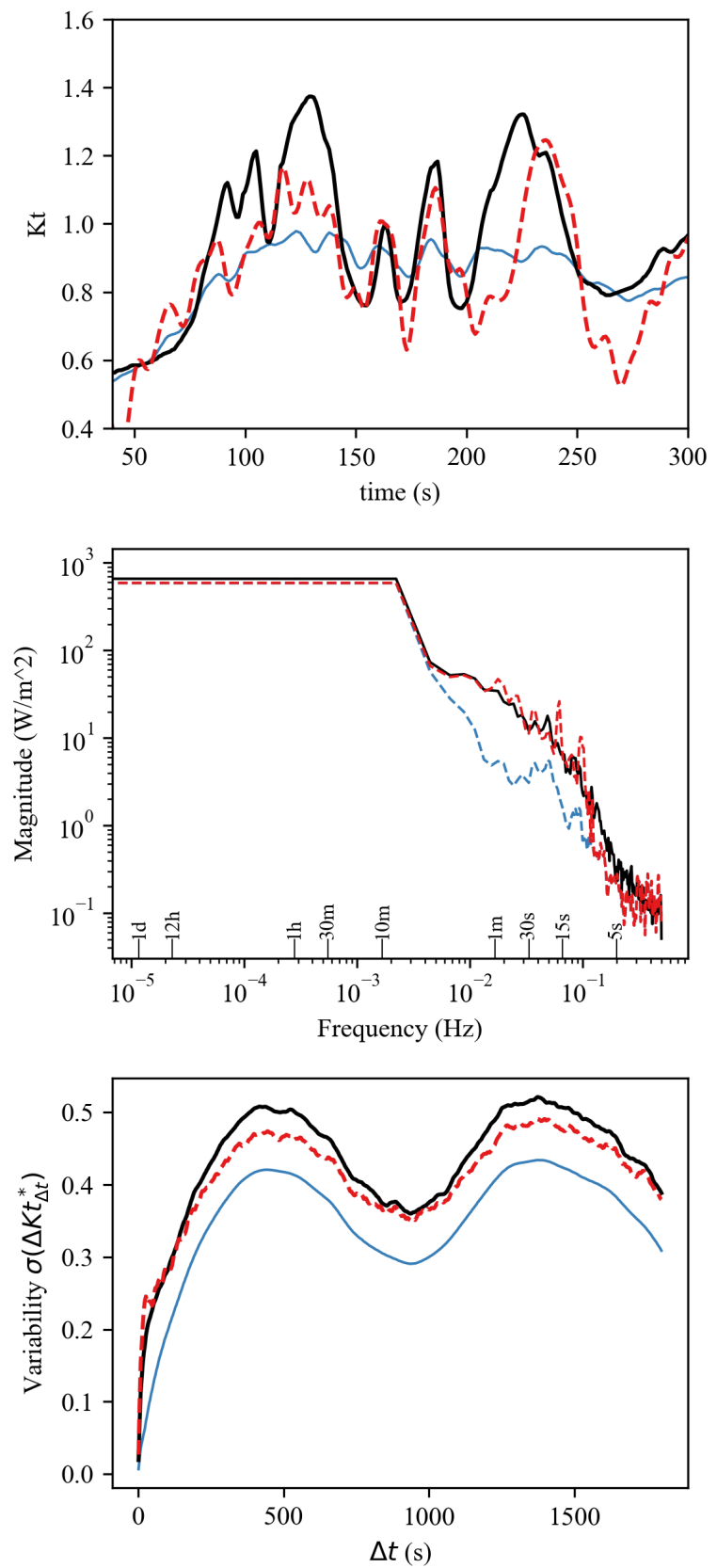

---- aggregate $\longrightarrow$ single site ---- CAM Single

Fig. 3. Disaggregation calculation time series, power spectrum and variability for 9 sites disaggregated to a single reference, with the low-power-spectrummagnitude correction applied. Spectra are smoothed using 8 averages with a Hanning window and $50 \%$ overlap. to this approach is that the results produced are deterministic in nature. That is, for a given plant and reference position within it, the transfer function, and therefore the disaggregated time series, will always be the same. There is the potential to marry this approach with other spatiotemporal variability techniques that instead highlight decorrelated and statistical forms of spatiotemporal variability, to incorporate all characteristics of variability in a complete model.

Given length requirements for the abstract, we attach the following note that for the full conference paper we hope to include comparison of results at multiple reference sites within the plant, and comparison with similar results utilizing the wavelet variability model (WVM), and a combination of the WVM and CAM.

\section{REFERENCES}

[1] R. Perez, M. David, T. E. Hoff, M. Jamaly, S. Kivalov, J. Kleissl, P. Lauret, and M. Perez, "Spatial and Temporal Variability of Solar Energy," Foundations and Trends in Renewable Energy, vol. 1, no. 1, pp. 1-44, Jul. 2016. [Online]. Available: https://www.nowpublishers.com/article/Details/REN-006

[2] G. M. Lohmann, "Irradiance Variability Quantification and Small-Scale Averaging in Space and Time: A Short Review," Atmosphere, vol. 9, no. 7, p. 264, Jul. 2018. [Online]. Available: https://doaj.org

[3] M. Lave, J. Kleissl, and J. S. Stein, "A Wavelet-Based Variability Model (WVM) for Solar PV Power Plants," IEEE Transactions on Sustainable Energy, vol. 4, no. 2, pp. 501-509, Apr. 2013.

[4] J. Marcos, I. d. 1. Parra, M. Garcia, and L. Marroyo, "Simulating the variability of dispersed large PV plants," Progress in Photovoltaics: Research and Applications, vol. 24, no. 5, pp. 680-691, 2016. [Online]. Available: https://onlinelibrary.wiley.com/doi/abs/10.1002/pip.2719

[5] J. Ranalli, E. E. Peerlings, and T. Schmidt, "Cloud Advection and Spatial Variability of Solar Irradiance." Virtual: IEEE, Jun. 2020, p. 8.

[6] A. Macke, P. Seifert, H. Baars, C. Barthlott, C. Beekmans, A. Behrendt, B. Bohn, M. Brueck, J. Bühl, S. Crewell, T. Damian, H. Deneke, S. Düsing, A. Foth, P. D. Girolamo, E. Hammann, R. Heinze, A. Hirsikko, J. Kalisch, N. Kalthoff, S. Kinne, M. Kohler, U. Löhnert, B. L. Madhavan, V. Maurer, S. K. Muppa, J. Schween, I. Serikov, H. Siebert, C. Simmer, F. Späth, S. Steinke, K. Träumner, S. Trömel, B. Wehner, A. Wieser, V. Wulfmeyer, and X. Xie, "The $\mathrm{HD}(\mathrm{CP})^{2}$ Observational Prototype Experiment (HOPE) - an overview," Atmospheric Chemistry and Physics, vol. 17, no. 7, pp. 4887-4914, Apr. 2017. [Online]. Available: https://www.atmos-chem-phys.net/17/4887/2017/acp-17-48872017-discussion.html 